\title{
Brown and brite adipocytes: same function, but different origin and response
}

\author{
Dinh-Toi Chu ${ }^{1,2,3 *}$, and Barbara Gawronska-Kozak, \\ 1. Institute for Research and Development, Duy Tan University, K7/25 Quang Trung, Danang, \\ Vietnam \\ 2. Centre for Molecular Medicine Norway (NCMM), Nordic EMBL Partnership, University of \\ Oslo and Oslo University Hospital, Norway \\ 3. Faculty of Biology, Hanoi National University of Education, Hanoi, Vietnam \\ 4. Institute of Animal Reproduction and Food Research, Polish Academy of Sciences, Olsztyn, \\ Poland
}

\section{*Correspondence to $\mathrm{BGK}$ :}

Institute of Animal Reproduction and Food Research, Polish Academy of Sciences, Olsztyn, Poland

Email: b.kozak@pan.olsztyn.pl

\section{Or DTC:}

Centre for Molecular Medicine Norway (NCMM), Nordic EMBL Partnership, University of Oslo and Oslo University Hospital, Norway, Gaustadalléen 21, 0349 Oslo, Norway

Email: chudinhtoi.hnue@gmail.com

\begin{abstract}
Inducing brown adipocytes in white adipose tissues is a promising target to combat obesity and its related disorders in human beings. This goal has been especially encouraged by new important discoveries of human brown adipose tissues. The accumulating evidence confirms the presence of active brown adipocytes, not only in newborns, but also in adult humans. In rodents, there are two populations of the Ucp1-expressing adipocytes with well characterizedthermogenic functions, classical interscapular brown adipocytes and brite/beige adipocytes (brown adipocytes that are induced in white adipose tissues). Importantly, the anatomical
\end{abstract}


localization, gene expression profiling and functional characterization of Ucp1-expressing fat cells indicates brite and brown adipocytes coexist in human beings. Therefore, the research directions of brown and brite adipogenesis provide lead to potential new therapies to fight obesity and its related metabolic diseases in human being. The objectives of this review are (1) to discuss the fate of primary adipocytes based on tissue origins, and (2) to discuss mechanisms of brown and brite adipogenesis which could lead to their different responses to browning reagents.

Keywords: brown adipocytes, brite adipocytes, adipocyte origin, adipogenesis

\section{Tissue origin determines adipocyte's destiny in vitro}

In mice, at least 17 types of fat depots carrying different adipocytes are recognized [1], and a major part of the scientific community classifies adipocytes in these depots into three categories: (i) classical brown, (ii) brite (inducible brown), and (iii) white fat cell [2]. Classical brown adipocytes share an origin of the myf5 positive $\left(\right.$ myf $\left.^{+}\right)$myotomal precursors with muscle cells [3, 4], while brite fat cells derive from myf5 negative (myf5) precursors [5, 6]. White adipocytes have both origins (myf5 ${ }^{+}$and myf5 precursors) (Reviewed in [2]). Long et al., in 2014 showed that brite adipocytes may share an origin with smooth muscles. They found that $5 \%$ of smooth muscle-like cells differentiated into brite adipocytes with requirement of PR domain containing 16 (Prdm16) in vitro and cold in vivo [7]. From a stem cell point of view, classical brown adipocytes arise from $\mathrm{myf}^{+}$precursors of mesenchymal stem cells (MSCs), brite adipocytes develop from myf5 myotomal precursors of MSCs, whereas white adipocytes derive from MSCs with myf5 $5^{-}$or myf5 $5^{+}$progenitors [2]. It is conceivable that isolation of stem cells from different anatomic locations of the body and type of precursors (myf5 $5^{-}$or myf5 $5^{+}$in a specific tissue could lead to differences in the type of primary adipocytes in vitro when they are stimulated to undergo adipogenic differentiation by the same conditions $[5,8]$. In other words, the adipogenic differentiation capacity of stem cells reflects the origin and characteristics of the fat depot. In vitro, cells isolated from the stromal vascular fraction (SVF) of interscapular brown adipose tissue (iBAT) cultured in adipogenic medium with rosiglitazone (Rosi) expressed high levels of classical brown adipocyte signatures (Uncoupling protein 1 - Ucp1, Zinc finger in the cerebellum 1 - Zicl, and Myogenin). On the contrary, cells that arise from stromal vascular fraction of epididymal fat depot (EPI) cultured in identical conditions show a fate of brite adipocytes with the specific markers (Ucpl and Homeo box C9 - Hoxc9). Both, Ucp1-expressing 
fat cells, have a full thermogenic program indicated by high expression of Peroxisome proliferator-activated receptor gamma coactivator 1-alpha (Pgcl $\alpha)$ and Peroxisome proliferator-activated receptor alpha (Ppara) [5]. Without Rosi in the adipogenic medium, EPI SVF develops into white adipocytes with the specific molecular markers such as Hocx8, Transcription factor 21 (Tcf21), Insulin-like growth factor-binding protein 3 (Igfbp3) and Dermatopontin (Dpt) [5]. These results suggest the presence of different types of precursor cells in iBAT and EPI that determine the direction of the adipogenic processes. iBAT has myf5 ${ }^{+}$ progenitors with the ability to develop into classical brown adipocytes. In contrast EPI has myf5 progenitors with the potential of differentiation into brite or white adipocytes.

Our published data [8] supports the statement that the tissue origin determines the fate of primary adipocytes in vitro. Under an identical adipogenic medium containing 3-isobutyl-1methylxanthine (IBMX), dexamethasone (DEX), insulin (INS) and Rosi, cells isolated from inguinal (ING) and iBAT fat tissues as well as ear mesenchymal stem cells (EMSC) differentiated robustly into adipocytes with significantly higher levels of the adipogenic marker (Ppar $\gamma)$ and abundant fat accumulation as indicated by oil red $\mathrm{O}$ staining [8]. Interestingly, Rosi induced three types of fat cells in vitro from three different tissues; brown adipocytes had arisen from iBAT, brite adipocytes were derived from ING, and white adipocytes were developed from EMSC as indicated by specific biofunctional markers [8]. Primary brown (iBAT) and brite adipocytes (ING) revealed a thermogenic program as certified by high expression of Ucpl, Pgcla and Ppara [8]. Primary white adipocytes differentiated from EMSC failed to express Ucp 1, Pgcl $\alpha$ and Ppara at both mRNA and protein levels [8]. Therefore, these adipocytes did not response to tested browning reagents including $\mathrm{NE}$ and $\mathrm{T} 3$ event alone or combined treatment (Fig. 7 in [8]).

\section{Mechanism of brown adipogenesis}

Brown adipose tissue (BAT) was first discovered in small adult mammals and in newborns where it functions as an adaptive organ to maintain body temperature in hypothermic conditions. Recently, the existence of brown adipose tissues and their thermogenic function in adult humans was recognized [9-14]. In rodents, the classical BAT is an interscapular brown adipose tissue, which is widely used to investigate the formation, development and function of brown adipocytes. Although brown and white fat cells display the opposite roles in lipid 
metabolism, they both require activity of transcription factor Ppary to be developed and active. In vivo, mice with a dominant-negative P465L mutation of Ppary have a defect in iBAT, but surprisingly not in white adipose tissue (WAT) [15].

Brown adipocytes differentiate from Myf5 expressing progenitor cells. The brown adipogenic differentiation needs coordinated action of two key transcription factors, Ppary and Early B-Cell Factor-2 (Ebf2) [16, 17]. The cooperation of these transcription factors promotes the expression and function of Prdm16, which then binds to Ppary and activates its transcriptional function to drive a brown adipocyte destiny [6]. Additionally, the thermogenic function of mature brown adipocytes is activated by $\beta$-adrenoreceptors' agonists, such as norepinephrine (NE) and epinephrine, which are released from sympathetic neurons [16] or adrenal glands. Active brown fat cells have a full thermogenic capacity with the expression and activities of important transcription factors as Ppara and Pgcla. The differentiation, development and function of brown fat cells are triggered by several factors including cold exposure, natriuretic peptides, thiazolidinediones, thyroid hormone (such as T3), bone morphogenetic protein 7 ( $\mathrm{Bmp} 7)$, bone morphogenetic protein $8 \mathrm{~b}(\mathrm{Bmp} 8 \mathrm{~b})$, fibroblast growth factor 21 (Fgf21) and orexin [16]. In vitro, the brown differentiation of iBAT SVF induced by a Ppary agonist is increased by a treatment of NE [5] or T3 [8]. T3 increases the thermogenesis of primary brown adipocytes from rat or hamster iBAT SVF by stimulating thyroid hormone nuclear T3 receptor beta 1 (TR $\beta 1)[18,19]$. In human, mRNA and protein levels of Ucpl in BAT SVF adipocytes are significantly elevated by a 4 hour-stimulation of NE [20].

The roles of some activators on brown adipogenesis were also indicated in our previous work where we examined the effects of $\mathrm{T} 3$ and NE on Rosi-stimulated brown adipogenesis of iBAT SVF (Fig. 7 in [8]). mRNA and protein analysis indicates that separate treatment with T3 or NE increased $U c p l$ expression induced by Rosi in primary brown adipocytes [8]. However, a combination of T3 and NE did not have a meaningful effect on brown adipogenesis. The Ucpl protein and mRNA levels of cells treated by $\mathrm{T} 3+\mathrm{NE}$ were not changed versus cells treated by $\mathrm{T} 3$ or NE alone [8].

\section{Mechanism of brite adipogenesis}


Inducible brown adipocytes or brite adipocytes (beige adipocytes, brown-like fat cells) were first described in rodents [21-23]. Initially these adipocytes were found in traditional white fat depots of rodents which were exposed to cold or injected by $\beta$ adrenergic receptor agonists [21-24]. Then, they were classified as a third type of adipocytes, brite adipocytes, beside classical brown and white fat cells. Brite adipocytes share the thermogenic function with brown adipocytes but they have a distinct developmental origin [5, 6, 24, 25]. Notably, there is the evidence of fully functional brite adipocytes in both infant and adult humans [14, 25-27]. But, our knowledge on origin and development of brite adipocytes is still limited.

Brite adipocytes come from non-Myf5 progenitor cells, but they share a common requirement for adipogenic differentiation with white and brown adipocytes that is the Ppary activation. In vitro, the Rosi stimulation induces brite adipocytes from epididymal white adipose tissue (EPI) SVF [5] and ING SVF [8] of mice. In vivo, cold exposure or treatment of $\beta$ adrenergic receptor by its agonists such as NE and CL (CL-316243, a B3-AR agonist) stimulates the formation of functionally active brite fat cells in white fat depots e.g. ING and EPI [21-23]. However, the induction of inducible brown adipocytes depends on several factors including genetics, nutritional conditions, developmental periods, environmental temperature and anatomic location of adipose tissues [28-30]. Stimulation of Ppary by its agonists induces brite adipogenesis by increasing the stability of Prdm16 and deacetylating Ppary followed by the recruitment of Prdm16 to Ppary target genes [16]. $\beta$ adrenergic receptor agonists and cold exposure enhance the expression and activity of $\operatorname{Pgcl\alpha }$, another key transcription factor in the development and function of brown fat cells [16]. Accordingly, the brite adipogenic differentiation is triggered by cold exposure, Rosi treatment, Fgf21, Irisin and natriuretic peptides [16, 31]. Fgf21 and Irisin have previously shown to be cold-induced endocrine activators of BAT function in humans [31]. Although sharing some general transcription factors for adipogenic differentiation with brown adipocytes including Ppary, Prdm16, Pgcl $\alpha$ and CCAAT/enhancer-binding protein beta $(C / e b p \beta)$, brite adipocytes behave in a different way than brown cells. During an adipogenic differentiation, T3 increases the expression of Ucpl protein and thermogenesis of iBAT SVF cultures, but this browning reagent does not affect thermogenesis and Ucp1 expression in WAT SVF cultures [19]. In addition, the 4-hour of NE stimulation in human primary cultures elevates both mRNA and protein levels of Ucp1 in primary brown but not in primary brite adipocytes [20]. 
In our previous works we also examined the brite adipogenesis of ING SVF, and their responses to the browning reagents, T3 and NE (Fig. 7 in [8]). Rosi stimulated brite adipocytes formation from ING with thermogenic program as indicated by remarkable expression of $U c p 1$, Pgcla, and Ppara genes [8]. The brite adipogenic effect of Rosi in ING SVF was not regulated by T3 or NE alone [8]. Interestingly, the combination of T3 and NE enhanced the Rosi-inducedadipogenic differentiation of brite fat cells as confirmed by elevated protein levels of UCP1 in Ao+NE+T3 versus Ao, Ao+NE or Ao+T3 treated cultures [8].

\section{Differences between brown and brite adipocytes in response to the browning reagents}

Brown and brite adipocytes arise from two different populations of precursor cells [3-6], but they share several features of development and function. Both kinds of $U c p l$ expressing fat cells need the activation of Ppary in vitro, or the stimulation of $\beta$ adrenergic receptors in vivo to trigger an adipogenic process [5, 8, 16, 21-23]. They perform thermogenic function by activity of Ucp1 in the association of other thermogenic transcription factors including Pgcl $\alpha$ and Ppara. However, experimental evidence shows that brown and brite adipocytes respond differently to NE and T3 under identical conditions [19, 20].

Our previous data also show dissimilar reactions of these Ucp1-expressing-adipocytes to $\mathrm{NE}$ and T3 when they were induced by Rosi in vitro [8]. As mentioned above, Rosi-stimulated brown, but not Rosi-stimulated brite adipogenenesis was enhanced by T3 or NE treatment alone [8] whereas, NE and T3 had a synergistic effect on brite, but not on brown fat cells triggered by the Rosi [8].

\section{Conclusions}

The accumulating evidence in the field suggests that the origin of progenitor cells determines the type of adipocytes in vitro, and brite and brown adipocytes are different in response to browning reagents. These were supported by our published data [8] summarized in Figure 1. Under the stimulation of Rosi in adipogenic medium (Ao), EMSC differentiated to white adipocytes as indicated by expression of white adipose tissue expansion markers such as Mest, while SVFs of ING and iBAT differentiated into brite and brown adipocytes respectively as proved by the expression of thermogenic genes such as Ucp1 (Fig. 5). In the same conditions brite and brown adipocytes responded differently to the stimulation of NE and T3. Brown 
adipocytes had increased thermogenic function by the single or combined treatments of these reagents, but brite adipocytes only responded to the combined stimulation of T3 and NE.

\section{Acknowledgments}

We would like to thank Dr. Leslie P. Kozak for critical reading and helpful comments to improve this manuscript before submitting.

The research in the Gawronska-Kozak laboratory is supported by grant from the National Science Centre, Poland (NCN, Poland) DEC-2012/05/B/NZ5/01537.

Dinh-Toi Chu is a postdoc under the SCIENTIA FELLOWS programme co-funded by Faculty of Medicine, University of Oslo and the EU Seventh Framework Programme (FP7) Marie S. Curie scheme - People: Cofunding of Regional, National and International Programmes (COFUND), grant agreement no. 609020.

\section{Conflict of interest}

The authors declare that there is no conflict of interest regarding the publication of this paper.

\section{References}

[1] Waldén T.B., Hansen I.R., Timmons J.A., Cannon B., and Nedergaard J. Recruited vs. nonrecruited molecular signatures of brown, "brite," and white adipose tissues. American Journal of Physiology Endocrinology And Metabolism 2011;302:E19-E31.

[2] Rosenwald M. and Wolfrum C. The origin and definition of brite versus white and classical brown adipocytes. Adipocyte 2014;3:4-9.

[3] Atit R., Sgaier S.K., Mohamed O.A., Taketo M.M., Dufort D., Joyner A.L., et al. $\beta$-catenin activation is necessary and sufficient to specify the dorsal dermal fate in the mouse. Developmental Biology 2006;296:164-176.

[4] Timmons J.A., Wennmalm K., Larsson O., Walden T.B., Lassmann T., Petrovic N., et al. Myogenic gene expression signature establishes that brown and white adipocytes originate from distinct cell lineages. Proceedings of the National Academy of Sciences 2007;104:4401-4406.

[5] Petrovic N., Walden T.B., Shabalina I.G., Timmons J.A., Cannon B., and Nedergaard J. Chronic Peroxisome Proliferator-activated Receptor $\gamma$ (PPAR $\gamma$ ) Activation of Epididymally Derived White Adipocyte Cultures Reveals a Population of Thermogenically Competent, UCP1-containing Adipocytes Molecularly Distinct from Classic Brown Adipocytes. Journal of Biological Chemistry 2010;285:71537164.

[6] Seale P., Bjork B., Yang W., Kajimura S., Chin S., Kuang S., et al. PRDM16 controls a brown fat/skeletal muscle switch. Nature 2008;454:961-967. 
[7] Long Jonathan Z., Svensson Katrin J., Tsai L., Zeng X., Roh Hyun C., Kong X., et al. A Smooth MuscleLike Origin for Beige Adipocytes. Cell metabolism 2014;19:810-820.

[8] Chu D.-T., Malinowska E., Gawronska-Kozak B., and Kozak L.P. Expression of Adipocyte Biomarkers in a Primary Cell Culture Models Reflects Preweaning Adipobiology. Journal of Biological Chemistry 2014;289:18478-18488.

[9] Cypess A.M., Lehman S., Williams G., Tal I., Rodman D., Goldfine A.B., et al. Identification and Importance of Brown Adipose Tissue in Adult Humans. New England Journal of Medicine 2009;360:1509-1517.

[10] Saito M., Okamatsu-Ogura Y., Matsushita M., Watanabe K., Yoneshiro T., Nio-Kobayashi J., et al. High Incidence of Metabolically Active Brown Adipose Tissue in Healthy Adult Humans: Effects of Cold Exposure and Adiposity. Diabetes 2009;58:1526-1531.

[11] Van Marken Lichtenbelt W.D., Vanhommerig J.W., Smulders N.M., Drossaerts J.M.A.F.L., Kemerink G.J., Bouvy N.D., et al. Cold-Activated Brown Adipose Tissue in Healthy Men. New England Journal of Medicine 2009;360:1500-1508.

[12] Virtanen K.A., Lidell M.E., Orava J., Heglind M., Westergren R., Niemi T., et al. Functional Brown Adipose Tissue in Healthy Adults. New England Journal of Medicine 2009;360:1518-1525.

[13] Zingaretti M.C., Crosta F., Vitali A., Guerrieri M., Frontini A., Cannon B., et al. The presence of UCP1 demonstrates that metabolically active adipose tissue in the neck of adult humans truly represents brown adipose tissue. The FASEB Journal 2009;23:3113-3120.

[14] Chu D.-T. and Tao Y. Human thermogenic adipocytes: a reflection on types of adipocyte, developmental origin, and potential application. Journal of Physiology and Biochemistry 2016;1-4.

[15] Sarah L. Gray, Edoardo Dalla Nora, and al e. Decreased Brown Adipocyte Recruitment and Thermogenic Capacity in Mice with Impaired Peroxisome Proliferator-Activated Receptor (P465L PPARy) Function. Endocrinology 2006;147:5708-5714.

[16] Harms M. and Seale P. Brown and beige fat: development, function and therapeutic potential. Nat Med 2013;19:1252-1263.

[17] Rajakumari S., Wu J., Ishibashi J., Lim H., Giang A., Won K., et al. EBF2 determines and maintains brown adipocyte identity. Cell Metab 2013;17:562 - 574.

[18] Martinez de Mena R., Scanlan T.S., and Obregon M.-J. The T3 Receptor $\beta 1$ Isoform Regulates UCP1 and D2 Deiodinase in Rat Brown Adipocytes. Endocrinology 2010;151:5074-5083.

[19] Klaus S., Ely M., Encke D., and Heldmaier G. Functional assessment of white and brown adipocyte development and energy metabolism in cell culture. Dissociation of terminal differentiation and thermogenesis in brown adipocytes. Journal of Cell Science 1995;108:3171-3180.

[20] Jespersen Naja Z., Larsen Therese J., Peijs L., Daugaard S., Homøe P., Loft A., et al. A Classical Brown Adipose Tissue mRNA Signature Partly Overlaps with Brite in the Supraclavicular Region of Adult Humans. Cell metabolism 2013;17:798-805.

[21] Lonçar D., Afzelius B.A., and Cannon B. Epididymal white adipose tissue after cold stress in rats I. Nonmitochondrial changes. Journal of Ultrastructure and Molecular Structure Research 1988;101:109-122.

[22] Guerra C., Koza R.A., Yamashita H., Walsh K., and Kozak L.P. Emergence of brown adipocytes in white fat in mice is under genetic control. Effects on body weight and adiposity. The Journal of Clinical Investigation 1998;102:412-420.

[23] Himms-Hagen J, J. C., Danforth E, J. T.D., Lang S. S, Waters B. L, et al. Effect of CL-316,243, a thermogenic beta 3-agonist, on energy balance and brown and white adipose tissues in rats. American Journal of Physiology - Regulatory, Integrative and Comparative Physiology 1994;266:R1371-R1382.

[24] Chu-Dinh T. and Chu D.T. 4-1BB and the Epigenetic Regulations of This Molecule. Medical Epigenetics 2014;2:80-85. 
[25] Wu J., Boström P., Sparks Lauren M., Ye L., Choi Jang H., Giang A.-H., et al. Beige Adipocytes Are a Distinct Type of Thermogenic Fat Cell in Mouse and Human. Cell 2012;150:366-376.

[26] Cypess A.M., White A.P., Vernochet C., Schulz T.J., Xue R., Sass C.A., et al. Anatomical localization, gene expression profiling and functional characterization of adult human neck brown fat. Nat Med 2013;19:635-639.

[27] Lidell M.E., Betz M.J., Leinhard O.D., Heglind M., Elander L., Slawik M., et al. Evidence for two types of brown adipose tissue in humans. Nat Med 2013;19:631-634.

[28] Kozak L.P., Koza R.A., Anunciado-Koza R., Mendoza T., and Newman S. Inherent Plasticity of Brown Adipogenesis in White Fat of Mice Allows for Recovery from Effects of Post-Natal Malnutrition. PLoS ONE 2012;7:e30392.

[29] Kozak L.P. The Genetics of Brown Adipocyte Induction in White Fat Depots. Frontiers in Endocrinology 2011;2:

[30] Xue B., Rim J.-S., Hogan J.C., Coulter A.A., Koza R.A., and Kozak L.P. Genetic variability affects the development of brown adipocytes in white fat but not in interscapular brown fat. Journal of Lipid Research 2007;48:41-51.

[31] Lee P., Linderman Joyce D., Smith S., Brychta Robert J., Wang J., Idelson C., et al. Irisin and FGF21 Are Cold-Induced Endocrine Activators of Brown Fat Function in Humans. Cell Metabolism 2014;19:302-309. 


\section{Figure Legends}

Figure 1. Schematic illustration of EMSC, ING and iBAT SVF cells response to adipogenic stimulation. Under the stimulation of Rosi in adipogenic medium (Ao), EMSC, ING and iBAT SVF cells differentiate into white, brite and brown adipocytes, respectively as indicated by the expression of specific markers: Mest for white, and Ucp1 for brite and brown adipocytes. Brite adipocytes developed from ING SVF cells, stimulated with NE+T3 but not NE or T3 alone have increase in Ucp1 expression. iBAT SVF cells differentiate into brown adipocytes responding to all browning reagents as indicated by increase in Ucp1 levels by either NE or T3 alone or combined treatment of NE and T3. 


\section{Figure 1.}

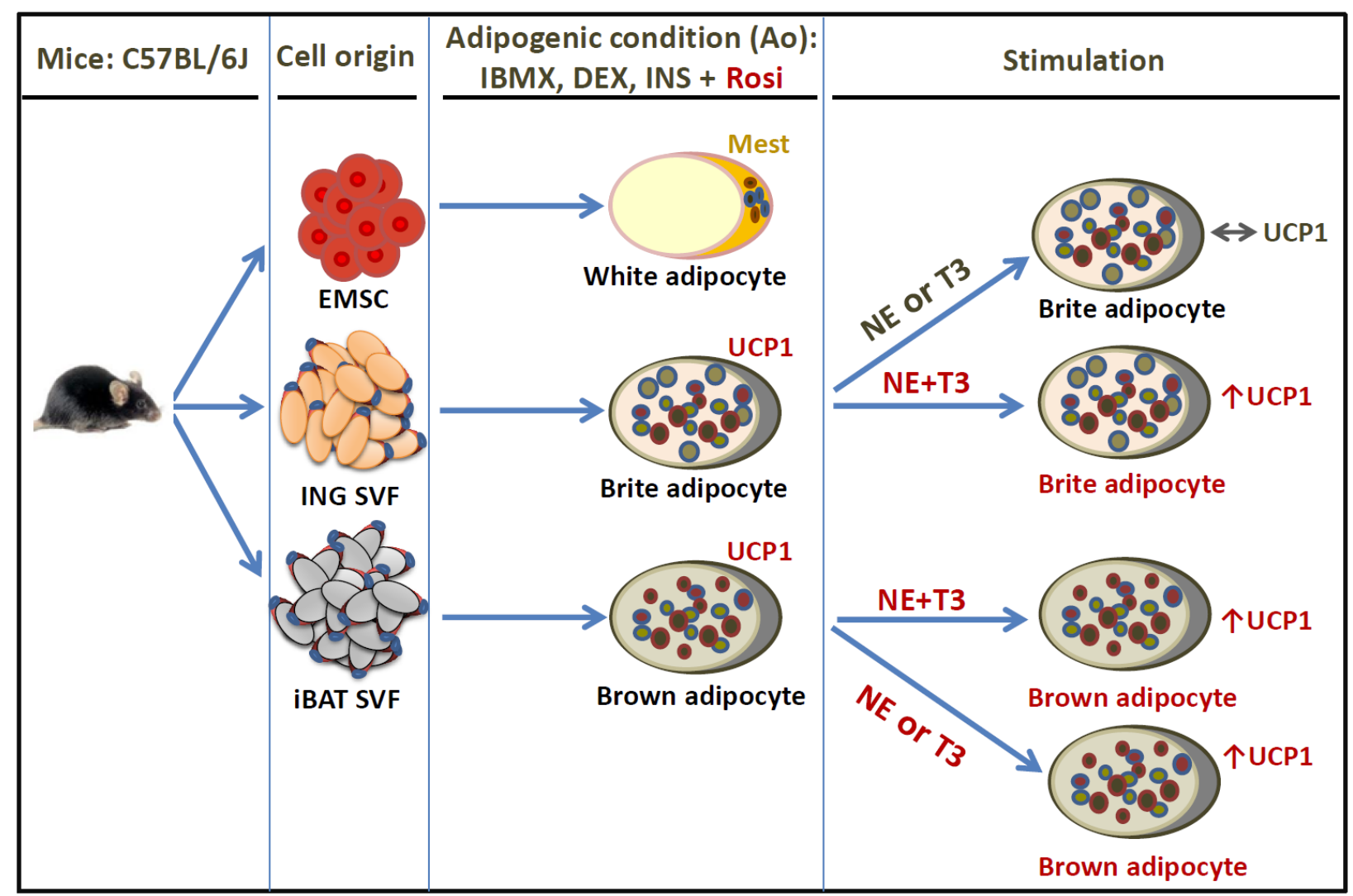

